\title{
What role for autologous marrow transplantation in cancer therapy?
}

\section{from Rainer Storb}

Toxicity to the marrow is a serious limitation of cancer therapy due to the vulnerability of the rapidly dividing haematopoietic stem cells to the anticancer agents. Higher - and potentially curative - doses of chemoradiotherapy may be possible when they are given in conjunction with replacement of the marrow by transplantation. However, because the donor marrow must be free of all tumour cells the graft has had to come from a separate, healthy individual raising all the problems of immunological compatibility and restricting treatment to those with suitable donors, either an identical twin or a histocompatible sibling. A way round this restriction would be possible if marrow could be taken from the affected individual before chemoradio therapy and cleared of tumour cells without destroying the haematopoietic cells. In this issue of Nature Krolick and his colleagues report some preliminary successes in mice using an antibody-toxin conjugate to purge marrow of tumour cells.

In general, for marrow grafting to be effective, two requirements must be fulfilled. First, tumours must be responsive to a short burst of high-dose therapy. Candidate tumours are leukemias and lymphomas, oat cell carcinoma of the lung, carcinoma of the breast, testis and ovary, soft-tissue sarcomas and pediatric malignancies such as neuroblastoma and Wilm's tumour. Second, as pointed out above, the infused marrow must be free of clonogenic tumour, for otherwise, tumour would recur from cells transferred with the marrow even when the body burden of tumour has been eradicated.

Much has been learned about tumour responsiveness from aggressive high-dose chemoradiotherapy of haematological malignancies using 'rescue' with healthy marrow either from an identical twin - a syngeneic graft - or from a sibling identical at the major histocompatibility complex - an HLA-identical allogeneic graft $^{1-5}$. Success has been most impressive in patients with acute nonlymphoblastic leukaemia (ANL) grafted in first remission, with a long-term disease-free survival rate of 55-60 per cent and relapse rates of 10 per cent after allogeneic grafts. The survival rate in patients with ANL grafted in relapse or second remission has been 25 per cent, with a relapse rate of 50 per cent. These rates compare with a survival rate of 20 per cent and a relapse rate of 70-80 per cent in patients with acute lymphoblastic leukaemia (ALL) grafted in

Rainer Storb is at the Fred Hutchinson Cancer Research Center, Seattle, Washington 98104. relapse or second remission. Results in non-Hodgkin's lymphoma are limited, but perhaps 50 per cent disease-free survival can be achieved. Relapse rates were significantly lower after allogeneic than after syngeneic grafts, perhaps because graft-versus-host disease contributed an antileukaemic effect ${ }^{6}$. Relapses originated almost exclusively in cells of host origin. Clearly, the problem of eradicating leukaemia has not yet been solved.

Nevertheless, the relative success of syngeneic and allogeneic marrow transplantation and the availability of effective cryopreservation techniques have stimulated research in autologous transplantation ${ }^{7}$ - transplantation of bone marrow within the individual. The major limitation in syngeneic and allogeneic transplantation is that only 25-40 per cent of all patients have suitable donors. The use of autologous marrow would extend high-dose therapy to larger groups of patients and avoid graft-versushost disease.

To achieve results with autologous marrow grafting comparable to those with syngeneic or allogeneic grafts the marrow must be cleaned of contaminating tumour cells without destroying haematopoietic stem cells. A number of laboratories have shown that this can be accomplished in certain rodent tumours using in vitro treatment with cytotoxic drugs or immune reagents. The latter have usually been absorbed antisera from a different species cytotoxic to the tumour but not to haematopoietic stem cells. In most experiments, haematopoietic cells were first contaminated with syngeneic tumour cells and then incubated with antibody and complement before infusion into a lethally irradiated syngeneic recipient. Successful elimination of murine $\mathrm{T}$ cell $\mathrm{AKR} / \mathrm{J}$ leukaemia and of, 6C3HED lymphoma with haematological restoration of the recipients have been reported ${ }^{8,9}$. Initial attempts have been described to apply in vitro treatment with antibody and complement to autologous human marrow grafts for common ALL ${ }^{10}$

Unfortunately, cell killing by antibody and complement is incomplete, and 5-10 per cent of tumour cells can be predicted to survive. Moreover, the quality of complement is variable. These problems with the conventional antibody plus complement approach emphasize the importance of the report by Krolick et al. (p.604) on the selective killing of leukaemia cells of the murine $\mathrm{B}$-cell tumour, $\mathrm{BCL}_{1}$, by antibody-toxin conjugates. They have treated $1-10 \times 10^{5}$ spleen cells from micebearing $\mathrm{BCL}_{1}, 80$ per cent of which were estimated to be tumour cells, with rabbit anti-mouse immunoglobulin antibody coupled with the A chain of the natural toxin ricin. From studies of cell transfers, they conclude that less than ten viable tumour cells remain in the spleen cell inocula. They then showed that antibodyricin treatment could be used to 'purge' marrow from $B C L_{1}$ tumour cells. Transplanted mice observed for 6 weeks did not show tumour although haematopoiesis was restored.

Encouraging as these results are, they must be interpreted with caution. First, the observation period of six weeks is short not only by human but also by murine standards. Leukaemic relapse has been seen as late as three and a half years after allogeneic human marrow grafting. Second, as the authors themselves point out, the $\mathrm{BCL}_{1}$ tumour consists almost entirely of cells expressing surface immunoglobulin and does not possess a major pre-B cell component, a quality perhaps not shared by human leukaemias.

Experimental animal tumours may be more mature because they are often perpetuated by way of passaging. In contrast, spontaneous human leukaemias may originate from stem cells closely resembling normal haematopoietic stem cells and not expressing the differentiation antigens characteristic of their leukaemic progeny. Indeed, studies using the marker glucose-6-phosphate dehydrogenase have suggested that ANL may involve stem cells capable of differentiating into granulocytes-monocytes, platelets and erythrocytes $^{11}$. Thus, despite complete removal of recognizable leukaemic cells from a marrow inoculum by antibodyricin conjugate, leukaemia might recur from infused leukaemic stem cells.

Despite these reservations, clinical studies should be undertaken. Monoclonal and polyvalent antibodies are now available that react with a variety of human tumour cells, including non-T cell ALL, T cell ALL, non-Hodgkin's lymphoma and $\mathrm{ANL}^{12}$. The results of syngeneic and allogeneic marrow transplantation will serve as the yardstick by which the potential of autologous transplantation can be measured.

1. Thomas, E.D. et al. New Engl. J. Med. 301, 597 (1979).

Johnson, F.L. et al. New Engl. J. Med. 305, 846 (1981)

. Fefer, A. et al. Blood 57, 421 (1981).

. Appelbaum, F.R. et al. Blood 58, 509 (1981).

5. Thomas, E.D. et al. New Engl. J. Med. 292, 832-895 (1975).

Weiden, P.L. et al. New Engl. J. Med. 300, 1068 (1979).

. Keystone Conf. on Autologous Bone Marrow Transplantation Expl. Hemat. 7, Suppl.5 (1979).

Thierfelder, S., Rodt, H. \& Netzel, B. Transplantation 23, 459 (1977).

9. Economou, J.S., Shin, H.S., Kaizer, H., Santos, G.W \& Schron, D.S. Proc Soc exp. Biol. Med. 158, 449 (1978).

10. Netzel, B., Haas, R.J., Rodt, H., Kolb, H.J.\& Thierfelder, S. Lancet i, 1330 (1980).

1. Fialkow, P.J. et al, Blood 57, 1068 (1981).

12. Ritz, J. \& Schlossman, S.F. Blood 59, I (1981) 